\title{
Patterns and predictors of sick leave after Covid-19 and long Covid in a national Swedish cohort
}

\author{
Emma Westerlind, Annie Palstam, Katharina S. Sunnerhagen and Hanna C. Persson*
}

\begin{abstract}
Background: The impact of Covid-19 and its long-term consequences is not yet fully understood. Sick leave can be seen as an indicator of health in a working age population, and the present study aimed to investigate sick-leave patterns after Covid-19, and potential factors predicting longer sick leave in hospitalised and non-hospitalised people with Covid-19.

Methods: The present study is a comprehensive national registry-based study in Sweden with a 4-month followup. All people who started to receive sickness benefits for Covid-19 during March 1 to August 31, 2020, were included. Predictors of sick leave $\geq 1$ month and long Covid ( $\geq 12$ weeks) were analysed with logistic regression in the total population and in separate models depending on inpatient care due to Covid-19.

Results: A total of 11,955 people started sick leave for Covid-19 within the inclusion period. The median sick leave was 35 days, 13.3\% were on sick leave for long Covid, and 9.0\% remained on sick leave for the whole follow-up period. There were 2960 people who received inpatient care due to Covid-19, which was the strongest predictor of longer sick leave. Sick leave the year prior to Covid-19 and older age also predicted longer sick leave. No clear pattern of socioeconomic factors was noted.

Conclusions: A substantial number of people are on sick leave due to Covid-19. Sick leave may be protracted, and sick leave for long Covid is quite common. The severity of Covid-19 (needing inpatient care), prior sick leave, and age all seem to predict the likelihood of longer sick leave. However, no socioeconomic factor could clearly predict longer sick leave, indicating the complexity of this condition. The group needing long sick leave after Covid-19 seems to be heterogeneous, indicating a knowledge gap.
\end{abstract}

Keywords: Covid-19, SARS-CoV2, Sick leave, Long Covid, Follow-up

\section{Background \\ Aim}

The aim of this study was to investigate patterns of sick leave, as well as factors predicting sick leave $\geq 1$ month and sick leave for long Covid in hospitalised as well as non-hospitalised persons with Covid-19, in a comprehensive national population.

\footnotetext{
* Correspondence: hanna.persson@neuro.gu.se

Department of Clinical Neuroscience, Institute of Neuroscience and Physiology, Sahlgrenska Academy, University of Gothenburg, and Sahlgrenska University Hospital, Per Dubbsgatan 14, 3 tr, 413 45, Gothenburg, Sweden
}

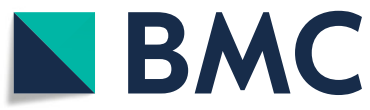

The Covid-19 pandemic affects the entire community and the long-term consequences are incalculable for the economy, public health, healthcare, and the health insurance system. Symptoms of Covid-19 vary, as do the severity and extent of functional impairment over time [1-6].

Sick leave can be seen as a complex indicator of wellbeing in the working-age population. The consequences of Covid-19 for working ability and sick leave are not yet known. A large cohort study comprising more than 1.6

(c) The Author(s). 2021 Open Access This article is licensed under a Creative Commons Attribution 4.0 International License, which permits use, sharing, adaptation, distribution and reproduction in any medium or format, as long as you give appropriate credit to the original author(s) and the source, provide a link to the Creative Commons licence, and indicate if changes were made. The images or other third party material in this article are included in the article's Creative Commons licence, unless indicated otherwise in a credit line to the material. If material is not included in the article's Creative Commons licence and your intended use is not permitted by statutory regulation or exceeds the permitted use, you will need to obtain permission directly from the copyright holder. To view a copy of this licence, visit http://creativecommons.org/licenses/by/4.0/ The Creative Commons Public Domain Dedication waiver (http://creativecommons.org/publicdomain/zero/1.0/) applies to the data made available in this article, unless otherwise stated in a credit line to the data. 
million workers in Spain compared total sick leave in the first trimester of 2020 with that in previous years and found that, overall, sick leave was $116 \%$ higher in March 2020, mostly related to infectious and respiratory disease [7]. The availability of paid sick leave varies among countries around the world [8], and in Sweden the financial compensation for sick leave is tax-funded and comprehensive. Furthermore, in Sweden the pandemic initially brought a rapid increase in sick leave, almost doubling the amount of sick leave during March and April 2020 compared with the previous year, according to the Swedish Social Insurance Agency (SSIA) [9]. The SSIA and the National Board of Health and Welfare in Sweden provide diagnosisspecific guidelines on sick leave and, since June 2020, for Covid-19. However, the guidelines for Covid-19 are vague due to a lack of knowledge of the prognosis of the disease-regardless of their need for inpatient care, an affected person's ability to work could decline in the aftermath [10].

It has been shown that symptoms related to Covid-19 can have a protracted course requiring intensive medical resources, whether or not the acute phase was critical [11-15]. Long Covid is a term describing this condition after the infection, with long-term symptoms such as fatigue, dyspnoea, pain, and depression [13-16]. The World Health Organization [16] defines long Covid as symptoms beyond 12 weeks. However, the cause, prevalence, duration, and prognosis of the protracted symptoms are still not clear [16].

\section{Methods}

\section{Settings and study population}

The present study is a registry-based study with data from the SSIA, the Swedish National Board of Health and Welfare, and Statistics Sweden, based on the unique Swedish personal identification number. It presents data on sick leave due to Covid-19 that started between March 1 and August 31, 2020, with follow-up for 4 months. A patient has been involved in the study process as a partner in research.

Inclusion criteria for the study population were the following: being registered with sickness benefits due to Covid-19, which were defined as the International Statistical Classification of Diseases (ICD) [17] code U07, starting within the inclusion period. Codes U (U00-U49) are used by WHO for provisional assignment of new diseases of uncertain etiology (Please see this link: https:// icd.who.int/browse10/2019/en\#/U07.1).

The SSIA is the public authority in Sweden that makes decisions on sick leave and pays sickness benefits, and it provided the study population and sick leave data for the present study. All working people in Sweden are eligible for sickness benefits from the SSIA if deemed to have reduced work ability due to sickness, regardless of citizenship or place of residence. Furthermore, selfemployment, parental leave, and unemployment (after previous employment) also entitle one to sickness benefits. The employer provides sick pay during the first 2 weeks of sickness absence; thereafter, the SSIA pays sickness benefits. If a person is unemployed, sickness benefits are provided by the SSIA from the start. In the present study, receiving sickness benefits regardless of amount is defined as sick leave.

The National Board of Health and Welfare provided data on date of death during the study period from the Cause of Death Register, which records all cases of death that have been registered in Sweden. Data from the $\mathrm{Na}$ tional Patient Register, which includes all inpatient care in Sweden, were used to investigate hospital stay due to Covid-19.

Statistics Sweden holds registries of all people registered in Sweden. Statistics Sweden provided data on sociodemographic variables to the study.

\section{Variables}

The sick-leave period in the present study includes at least one period of sickness benefits due to Covid-19 diagnosis. Other predefined related diagnoses were merged with the Covid-19 sick leave if the gap of nonregistration between sick leaves was $\leq 2$ weeks. The related diagnoses are shown in additional Table 1 and included, for example, unspecified virus infections, fever, and postviral fatigue syndrome, but also a second sickleave registration for Covid-19 diagnosis. In cases of sick pay provided by the employer, these were also merged with the sick-leave period. The sick-leave period could comprise a maximum of 122 days (4 months of followup). For predictive analyses, sick leave was dichotomised into sick leave $\geq 1$ month ( $\geq 30$ days): yes/no, and sick leave for long Covid ( $\geq 12$ weeks, in line with the WHO definition [16]): yes/no.

Sick leave prior to Covid-19 was defined as either being on sick leave for one period of at least 28 days between March 1, 2019, and the date of first Covid-19 sick leave registration, or being on sick leave at least six times during the same period of time.

The SSIA register includes the employment status for which a person receives the sick leave. The types of employment comprise employment (including parental leave, and combined employment and self-employment), selfemployment, and unemployment (including studies).

Educational level was categorised as primary school ( $\leq$ 9 years), secondary school (10-12 years), short university education (13-14 years), or long university education ( $\geq 15$ years). The educational level registered in 2019 was used. The income variable was the disposable income for each person during 2019, presented in thousands of 
Swedish krona SEK (1 Euro=10.16 SEK, March 4, 2021). Income was categorised in tertiles of low, medium, and high income. Country of birth was presented as Sweden, Nordic countries except for Sweden, European countries except for the Nordic countries, and Countries outside of Europe. For marital status in 2020, married and registered partnership were both classified as married. Likewise, divorced and widow/widowed meant a change from either marriage or registered partnership. Inpatient care due to Covid-19 was classified as being registered with a hospital stay of $>1$ day with a registration of any of the Covid-19 diagnoses U07. The primary diagnoses are presented in additional Table 2 in the cases where U07 was not registered as the primary diagnosis.

\section{Statistical methods}

The data were processed and analysed using IBM SPSS Statistics 25. Data are presented as number and percentage (\%), mean and standard deviation (SD), and median and interquartile range (IQR). The significance level (alpha) was set to 5\%. To compare differences between groups, the Mann-Whitney U test and Fisher exact test were used.

To graphically present cumulative incidence of sick leave over time, Kaplan-Meier curves were used. There was no censoring, as cases of death during the study period were treated with a worst-case-scenario approach and were set at sick leave for the maximum number of days, and there was no other loss to follow-up.

Multiple logistic regression was used for predictive analysis. The regression analyses were performed on three separate populations: the total study population, the participants receiving inpatient care for Covid-19, and the participants not receiving inpatient care. Two different dependent variables were used in different models: sick leave $\geq 1$ month and sick leave for long Covid. The independent variables were chosen based on clinical and theoretical reasoning: age, sex, educational level, income, country of birth, sick leave prior to Covid-19, employment status, marital status, and inpatient care due to Covid-19. The results are presented as odds ratio (OR), 95\% confidence interval $(95 \% \mathrm{CI})$, and $p$-value in forest plots. The ordinal or continuous independent variables were tested for multicollinearity using the Spearman correlation test, with values $<0.3$ being acceptable. To test the accuracy of the models, receiver operating characteristics (ROC) curves were constructed. An area under the ROC curve > 0.70 indicate acceptable accuracy [18].

\section{Results}

\section{Study population}

The study population consists of 11,955 included participants. Of these, 7983 (66.8\%) were registered with sick leave due to" Covid-19, virus detected", 3949 (33.0\%) as" Covid-19, virus undetected", and $23(0.2 \%)$ as "unspecified Covid-19 diagnosis". As presented in Tables 1, 2960 (24.8\%) received inpatient care due to Covid-19 during the study period. Women comprised $60 \%$ of the total study population $(n=7129)$, whereas men were $64 \%$ $(n=1894)$ of the participants receiving inpatient care due to Covid-19.

A total of 24 participants $(0.2 \%)$ died within the study period, all within $\leq 9$ days from the end of the sick-leave period.

\section{Sick-leave patterns}

Of the participants, 1931 (16.2\%) were on sick leave for at least $28 \mathrm{~d}$ or 6 times the year prior to the Covid-19 infection.

The median duration of sick leave due to Covid-19 was 35 days (IQR 26, mean 47.5, SD 29.96). There were 7903 (66.1\%) participants on sick leave $\geq 1$ month, and $1073(9.0 \%)$ continued their sick leave during the whole follow-up period, that is, for at least 4 months (Fig. 1).

A total of 1592 (13.3\%) were on sick leave for at least 12 weeks, and thus defined as having long Covid. The participants on sick leave for long Covid were significantly older $(p<0.001)$, predominantly men $(p<0.001)$, spent more time on sick leave prior to Covid-19 $(p<$ $0.001)$, and were more likely to have received inpatient care $(p<0.001)$ than the participants not on sick leave for long Covid.

\section{Predictors of sick leave}

Predictors of sick leave $\geq 1$ month, and predictors of sick leave for long Covid, are presented in Fig. 2. Older age, sick leave prior to Covid-19, and inpatient care due to Covid-19 resulted in significantly higher odds for being on sick leave with both outcomes. Different socioeconomic factors were significant for sick leave $\geq 1$ month and sick leave for long Covid, respectively.

For the participants receiving inpatient care due to Covid-19, older age meant significantly higher odds, and higher income significantly lower odds, of sick leave both $\geq 1$ month and for long Covid (Fig. 3). Other variables differed between the models.

For the participants not receiving inpatient care due to Covid-19, sick leave prior to Covid-19 resulted in significantly higher odds of sick leave in both outcomes (Fig. 4). Age, country of birth, employment status, and marital status all gave different odds of sick leave $\geq 1$ month, and sick leave for long Covid, respectively.

\section{Discussion}

In this registry-based national cohort, the median duration of sickness benefits due to Covid-19 was more than 1 month (35 days). Furthermore, more than one out 
Table 1 Characteristics of the participants

\begin{tabular}{|c|c|c|c|}
\hline & Total & Inpatient care & No inpatient care \\
\hline Participants, n (\%) & $11,955(100.0)$ & $2960(24.8)$ & $8995(75.2)$ \\
\hline \multicolumn{4}{|l|}{ Sex, n (\%) } \\
\hline Men & $4826(40.4)$ & $1894(64.0)$ & $2932(32.6)$ \\
\hline Women & $7129(59.6)$ & $1066(36.0)$ & $6063(67.4)$ \\
\hline Age, mean (SD) & $48.0(11.3)$ & $52.0(9.9)$ & $46.7(11.4)$ \\
\hline \multicolumn{4}{|l|}{ Country of birth, $\mathrm{n}(\%)^{\mathrm{a}}$} \\
\hline Sweden & $7545(63.1)$ & $1558(52.7)$ & $5987(66.6)$ \\
\hline Nordic countries except for Sweden & $271(2.3)$ & $85(2.9)$ & $186(2.1)$ \\
\hline European countries except for the Nordic countries & $1210(10.1)$ & $370(12.5)$ & $840(9.3)$ \\
\hline Countries outside of Europe & $2929(24.5)$ & $944(31.9)$ & $1976(22.9)$ \\
\hline \multicolumn{4}{|l|}{ Educational level, n (\%) } \\
\hline Primary school ( $\leq 9$ years) & $1237(10.4)$ & $411(14.0)$ & $826(9.2)$ \\
\hline Secondary school (10-12 years) & $5889(49.6)$ & $1406(47.9)$ & $4483(50.2)$ \\
\hline Short university education (13-14 years) & $1743(14.7)$ & $466(15.9)$ & $1277(14.3)$ \\
\hline Long university education ( $\geq 15$ years) & $3995(25.2)$ & $650(22.2)$ & $2345(26.3)$ \\
\hline \multicolumn{4}{|l|}{ Income, 1000 SEK median (IQR) ${ }^{c}$} \\
\hline Low income & $212(61)$ & $213(66)$ & $211(60)$ \\
\hline Medium income & $288(33)$ & $290(33)$ & $287(33)$ \\
\hline High income & $390(100)$ & $404(121)$ & $384(94)$ \\
\hline \multicolumn{4}{|l|}{ Marital status, n (\%) } \\
\hline Married & $5812(48.8)$ & $1695(57.8)$ & $4117(45.8)$ \\
\hline Single & $3859(32.4)$ & $671(22.9)$ & $3188(35.5)$ \\
\hline Divorced & $2097(17.6)$ & $536(18.3)$ & $1561(17.4)$ \\
\hline Widow/Widower & $149(1.3)$ & $31(1.1)$ & $118(1.3)$ \\
\hline \multicolumn{4}{|l|}{ Sick leave prior to Covid-19, n (\%) } \\
\hline Sick leave $\geq 28$ days & $1918(16.0)$ & $357(12.1)$ & $1561(17.4)$ \\
\hline Sick leave $\geq 6$ times & $26(0.2)$ & $5(0.2)$ & $21(0.2)$ \\
\hline \multicolumn{4}{|l|}{ Employment status, n (\%) ${ }^{f}$} \\
\hline Employment & $11,460(95.9)$ & $2756(93.1)$ & $8704(96.8)$ \\
\hline Self-employment & $288(2.4)$ & $105(3.5)$ & $183(2.0)$ \\
\hline Unemployment & $204(1.7)$ & $99(3.3)$ & $105(1.2)$ \\
\hline
\end{tabular}

$a=9$ missing, $b=91$ missing, $c=2$ missing, $d=38$ missing, $e=13$ participants on sick leave both $\geq 28$ days and $\geq 6$ times, $f=3$ missing. Abbreviations: $S D$, standard deviation; IQR: interquartile range.

of ten subjects were on sick leave for more than 12 weeks, defined as long Covid. Sick leave due to Covid-19 is an unexplored area, and increased knowledge is essential for a national and global perspective. The results from this study show that Covid-19 places a large burden not only on affected people and the health-care system, but also on the health insurance system. A large proportion of people with Covid-19 had a protracted course of sick leave, in both the present study and a published preprint [12], and a long-term perspective is needed for these patients. The actual prevalence of long Covid is unclear both globally and in Sweden [16], but the present study suggests that it affects a substantial proportion of patients, as $13 \%$ of the people with sickness benefit due to Covid-19 were on sick leave for long Covid. To the best of our knowledge, this is the first study to estimate long Covid and its consequences from sick-leave data. In a preprint study [12] using selfreported symptoms as a measure of long Covid, the majority reported that their ability to work was reduced for several months after Covid-19, but $27 \%$ were working as before. This indicates that the actual prevalence of long Covid is even higher in Sweden than the number on sick leave for long Covid presented in this study.

The need for inpatient care due to Covid-19 was a significant predictor of sick leave $\geq 1$ month, and it 


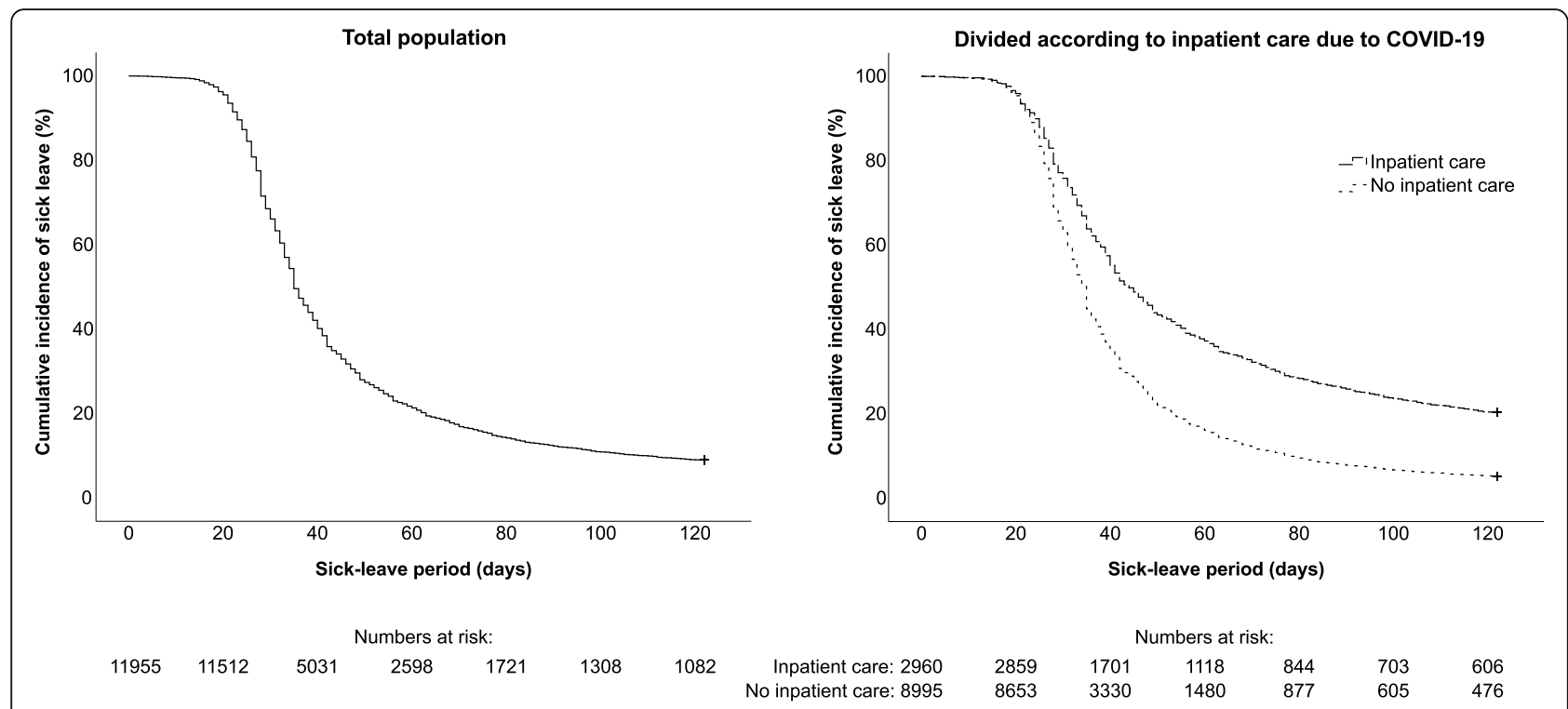

Fig. 1 Cumulative incidence of sick leave during the study period. Total study population (left) and divided according to inpatient care due to Covid-19 (right)

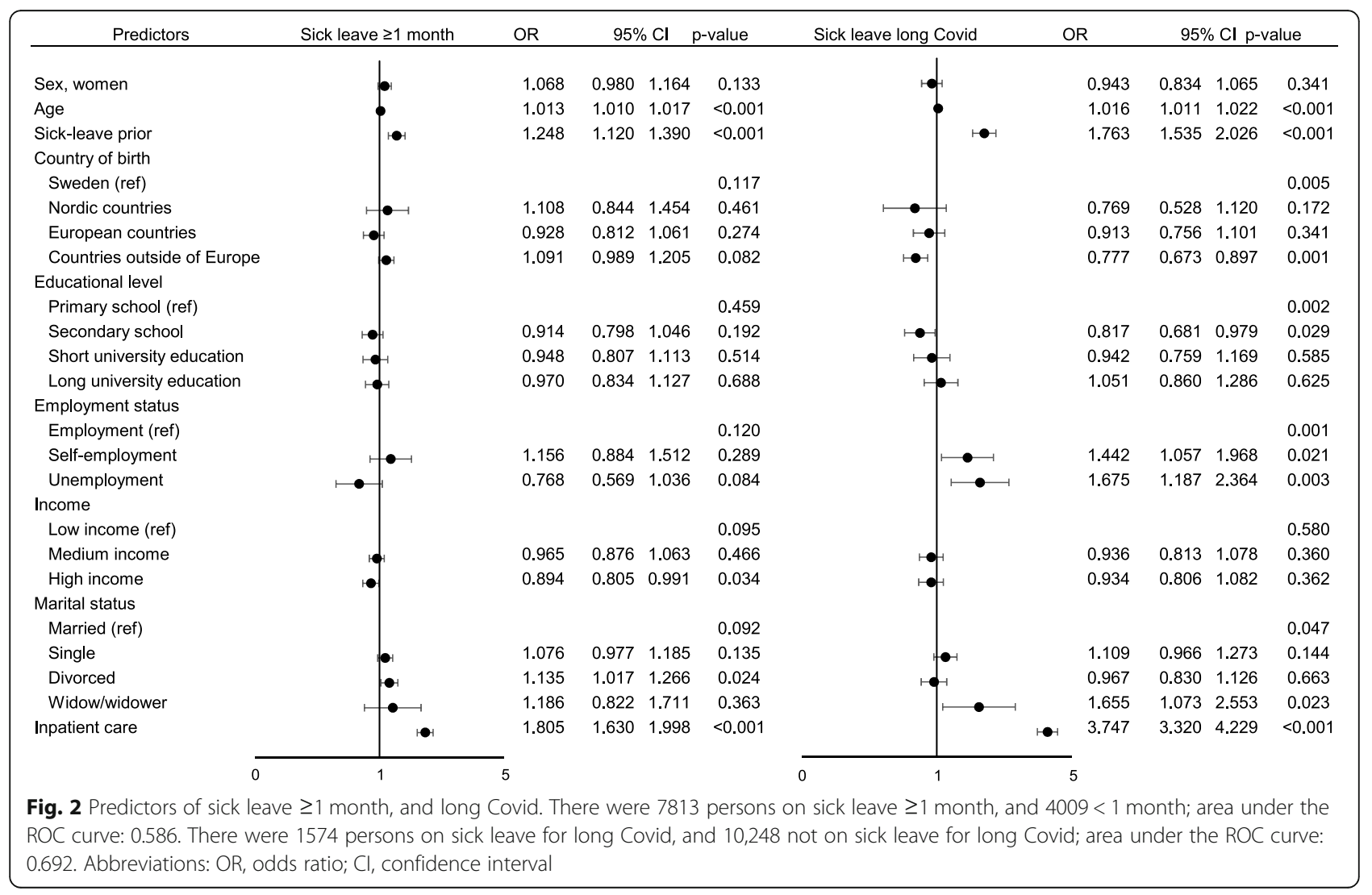




\begin{tabular}{|c|c|c|c|c|c|c|c|c|c|c|}
\hline \multirow{2}{*}{$\begin{array}{l}\text { Predictors } \\
\text { Sex, women }\end{array}$} & \multirow{2}{*}{ Sick leave $\geq 1$ month } & \multirow{2}{*}{$\begin{array}{l}\text { OR } \\
1.176\end{array}$} & \multicolumn{2}{|c|}{$95 \% \mathrm{Cl}$} & \multirow{2}{*}{$\frac{p \text {-value }}{0.097}$} & Sick leave long Covid & OR & \multicolumn{3}{|c|}{$95 \% \mathrm{Cl} \mathrm{p}$-value } \\
\hline & & & 0.971 & 1.424 & & $\bullet \bullet-1$ & 0.737 & 0.612 & 0.887 & 0.001 \\
\hline Age & & 1.030 & 1.021 & 1.040 & $<0.001$ & & 1.031 & 1.021 & 1.041 & $<0.001$ \\
\hline Sick-leave prior & $\bullet-1$ & 1.198 & 0.904 & 1.587 & 0.209 & $\bullet-1$ & 1.777 & 1.396 & 2.262 & $<0.001$ \\
\hline \multicolumn{11}{|l|}{ Country of birth } \\
\hline Sweden (ref) & & & & & 0.836 & & & & & 0.412 \\
\hline Nordic countries & $\longmapsto$ & 1.223 & 0.686 & 2.178 & 0.495 & $\longmapsto$ & 0.881 & 0.533 & 1.457 & 0.623 \\
\hline European countries & $\mapsto$ & 0.925 & 0.700 & 1.221 & 0.581 & $\mapsto$ & 0.956 & 0.730 & 1.252 & 0.744 \\
\hline Countries outside of Europe & $\mapsto$ & 0.979 & 0.794 & 1.207 & 0.842 & $\bullet \bullet$ & 0.840 & 0.683 & 1.033 & 0.098 \\
\hline \multicolumn{11}{|l|}{ Educational level } \\
\hline Primary school (ref) & & & & & 0.268 & & & & & 0.509 \\
\hline Secondary school & $\mapsto$ & 1.007 & 0.768 & 1.320 & 0.961 & $\mapsto$ & 0.947 & 0.734 & 1.222 & 0.677 \\
\hline Short university education & 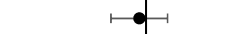 & 0.927 & 0.672 & 1.279 & 0.645 & $\bullet$ & 0.808 & 0.591 & 1.106 & 0.184 \\
\hline Long university education & $\bullet$ & 0.808 & 0.598 & 1.093 & 0.167 & $\rightarrow$ & 0.976 & 0.727 & 1.310 & 0.870 \\
\hline \multicolumn{11}{|l|}{ Employment status } \\
\hline Employment (ref) & & & & & 0.694 & & & & & 0.001 \\
\hline Self-employment & $\longmapsto \bullet$ & 1.226 & 0.736 & 2.043 & 0.434 & $\bullet$ & 1.292 & 0.838 & 1.993 & 0.247 \\
\hline Unemployment & $\longmapsto$ & 0.930 & 0.569 & 1.519 & 0.772 & $\longmapsto$ & 2.184 & 1.424 & 3.348 & $<0.001$ \\
\hline \multicolumn{11}{|l|}{ Income } \\
\hline Low income (ref) & & & & & 0.006 & & & & & 0.024 \\
\hline Medium income & -1 & 1.122 & 0.882 & 1.427 & 0.348 & $\mapsto$ & 1.018 & 0.815 & 1.272 & 0.876 \\
\hline High income & $\vdash \bullet$ & 0.791 & 0.627 & 0.998 & 0.048 & $\bullet$ & 0.778 & 0.620 & 0.978 & 0.031 \\
\hline \multicolumn{11}{|l|}{ Marital status } \\
\hline Married (ref) & & & & & 0.900 & & & & & 0.257 \\
\hline Single & $b-1$ & 1.092 & 0.871 & 1.369 & 0.447 & - -1 & 1.190 & 0.956 & 1.481 & 0.120 \\
\hline Divorced & 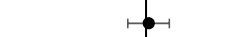 & 1.030 & 0.813 & 1.304 & 0.806 & $\mapsto$ & 0.902 & 0.719 & 1.133 & 0.377 \\
\hline \multirow[t]{2}{*}{ Widow/widower } & $\longrightarrow$ & 1.016 & 0.410 & 2.519 & 0.973 & $b$ & 1.038 & 0.468 & 2.303 & 0.927 \\
\hline & 1 & 5 & & & & 1 & 5 & & & \\
\hline
\end{tabular}

\begin{tabular}{|c|c|c|c|c|c|c|c|c|c|c|}
\hline \multirow{2}{*}{$\begin{array}{l}\text { Predictors } \\
\text { Sex, women }\end{array}$} & \multirow{2}{*}{ Sick leave $\geq 1$ month } & \multirow{2}{*}{$\begin{array}{l}\mathrm{OR} \\
1.048\end{array}$} & \multicolumn{2}{|c|}{$95 \% \mathrm{Cl}$} & \multirow{2}{*}{$\begin{array}{l}\mathrm{p} \text {-value } \\
\\
+\quad 0.345\end{array}$} & \multirow{2}{*}{ Sick leave long Covid } & \multirow{2}{*}{$\begin{array}{l}\text { OR } \\
1.173\end{array}$} & \multicolumn{3}{|c|}{$95 \% \mathrm{Cl}$ p-value } \\
\hline & & & 0.951 & 1.154 & & & & 0.989 & 1.391 & 0.067 \\
\hline Age & & 1.010 & 1.006 & 1.014 & $+<0.001$ & & 1.007 & 1.000 & 1.014 & 0.067 \\
\hline Sick-leave prior & 1曰-1 & 1.256 & 1.117 & 1.412 & $2<0.001$ & $\bullet \bullet-1$ & 1.794 & 1.512 & 2.129 & $<0.001$ \\
\hline \multicolumn{11}{|l|}{ Country of birth } \\
\hline Sweden (ref) & & & & & 0.074 & & & & & 0.006 \\
\hline Nordic countries & $\mapsto$ & 1.055 & 0.773 & 1.441 & 0.736 & $\bullet$ & 0.634 & 0.349 & 1.150 & 0.134 \\
\hline European countries & & 0.924 & 0.792 & 1.077 & 0.311 & $\bullet$ & 0.842 & 0.644 & 1.102 & 0.210 \\
\hline Countries outside of Europe & $\bullet$ & 1.131 & 1.010 & 1.266 & 0.032 & $\bullet-1$ & 0.714 & 0.581 & 0.878 & 0.001 \\
\hline \multicolumn{11}{|l|}{ Educational level } \\
\hline Primary school (ref) & & & & & 0.119 & & & & & $<0.001$ \\
\hline Secondary school & - & 0.895 & 0.764 & 1.048 & 30.168 & $\bullet$ & 0.723 & 0.554 & 0.942 & 0.016 \\
\hline Short university education & de & 0.959 & 0.797 & 1.155 & $5 \quad 0.662$ & $\longrightarrow$ & 1.062 & 0.785 & 1.437 & 0.696 \\
\hline Long university education & - & 1.012 & 0.850 & 1.205 & $5 \quad 0.893$ & $\mapsto$ & 1.050 & 0.791 & 1.395 & 0.734 \\
\hline \multicolumn{11}{|l|}{ Employment status } \\
\hline Employment (ref) & & & & & 0.071 & & & & & 0.096 \\
\hline Self-employment & 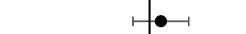 & 1.138 & 0.828 & 1.564 & 0.425 & $\bullet$ & 1.596 & 1.015 & 2.511 & 0.043 \\
\hline Unemployment & $\bullet$ & 0.652 & 0.441 & 0.965 & $5 \quad 0.032$ & $\longmapsto$ & 0.750 & 0.345 & 1.632 & 0.468 \\
\hline \multicolumn{11}{|l|}{ Income } \\
\hline Low income (ref) & & & & & 0.394 & & & & & 0.040 \\
\hline Medium income & & 0.938 & 0.844 & 1.043 & 0.239 & $\bullet \bullet$ & 0.873 & 0.725 & 1.052 & 0.155 \\
\hline High income & . & 0.931 & 0.828 & 1.047 & 0.234 & - -1 & 1.114 & 0.917 & 1.354 & 0.278 \\
\hline \multicolumn{11}{|l|}{ Marital status } \\
\hline Married (ref) & & & & & 0.077 & & & & & 0.027 \\
\hline Single & r & 1.068 & 0.960 & 1.189 & 0.226 & b-1 & 1.071 & 0.893 & 1.284 & 0.459 \\
\hline Divorced & 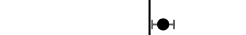 & 1.167 & 1.031 & 1.322 & 0.015 & -1 & 1.023 & 0.830 & 1.260 & 0.832 \\
\hline \multirow[t]{2}{*}{ Widow/widower } & $\mapsto$ & 1.224 & 0.819 & 1.830 & 0.325 & & 2.143 & 1.296 & 3.543 & 0.003 \\
\hline & 1 & 5 & & & & 1 & 5 & & & \\
\hline \multicolumn{11}{|c|}{$\begin{array}{l}\text { Fig. } 4 \text { Predictors of sick leave } \geq 1 \text { month, and long Covid, for participants' not receiving inpatient care. There were } 5611 \text { persons on sick leave } \geq 1 \\
\text { month, and } 3305<1 \text { month; area under the ROC-curve: } 0.550 \text {. There were } 789 \text { persons on sick leave for long Covid, and } 8127 \text { not on sick leave } \\
\text { for long Covid; area under the ROC-curve: 0.609. Abbreviations: OR, odds ratio; Cl, confidence interval }\end{array}$} \\
\hline
\end{tabular}


increased by more than three-fold the odds of needing sick leave for long Covid compared with no inpatient care due to Covid-19. It is likely that a patient who requires extensive care due to critical Covid-19 illness will have restrictions in function for a long time [19]. Five years after being treated at an intensive care unit for acute respiratory distress syndrome, people still had decreased levels of physical function [20]. In line with the present findings, hospitalisation has also been shown to predict protracted symptoms after Covid-19 [11], and long-term symptoms exist in both hospitalised and non-hospitalised Covid-19 patients in a preprint study [13]. People suffering from long Covid seem to be a heterogeneous group [13], including both those who have been critically ill in the acute phase and those with non-critical illness, and it may be speculated that the consequences of Covid-19 differ in the two groups. Possible sex differences are one factor to consider.

Prior studies have repeatedly reported that men have more severe Covid-19, with higher mortality, a need for more medical care, and worse outcomes [21]. In this study, in line with previous research, the patients who received inpatient care due to Covid-19 were mostly men (64\%). Furthermore, the proportion of men was significantly higher than the proportion on women in the group on sick leave for long Covid compared with the group not on sick leave for long Covid. In addition, men had higher odds of longer sick leave for Covid-19, both in the total population and in the population receiving inpatient care due to Covid-19. However, the present study comprised all people who received sickness benefits due to Covid-19 in Sweden, of whom a clear majority $(60 \%)$ were women. This can be compared with the number of confirmed cases of Covid-19 in Sweden, where women represent $52 \%$ of the cases [22]. Furthermore, in absolute numbers, there were more women than men in the long-Covid group. This is in line with previous data showing that women generally receive more sick leave in Sweden [9] and have lower odds of returning to work after various diagnoses [23, 24]. The patients receiving sick leave for long Covid seem to be a heterogeneous group, with both critical and non-critical acute phases, and it is important not to neglect any of them.

To be on sick leave for more than 28 days or more than six times in the year before having Covid-19 resulted in higher odds of sick leave $\geq 1$ month and for long Covid in most of the analyses. This could reflect higher comorbidity in the people with prior sick leave, or a higher vulnerability for sick leave in general. Other studies have shown that there is an association between previous sick leave and sick leave after stroke [25], traumatic brain injury [26], and mental disorder [27], and the present study indicates this also true for Covid-19.
Older age predicted sick leave $\geq 1$ month and for long Covid in most of the analyses. Older age is highly associated with worse outcome after Covid-19 [28]. Furthermore, it can be speculated that health-care providers, the SSIA, and employers all act to optimise the return to work for younger people.

The pandemic seems to strike differently depending on socioeconomic or ethical conditions, both globally $[29,30]$ and in Sweden [31]. Various socioeconomic factors predicted longer sick leave for Covid-19 in this study, but the results are not clear and consistent throughout the models. To get the whole picture of factors affecting post-Covid-19 outcomes, and specifically sick leave, more research is needed. Perhaps more workrelated predictors have to be included, or types of persistent symptoms. Our results did not yield distinct predictions for hospitalised vs. non-hospitalised people. Perhaps other subgroups within the heterogeneous group of persons requiring long-term sick leave after Covid-19 need to be analysed in order to paint a clearer picture.

\section{Methodology discussion and study limitations}

The present cohort included only people receiving sickness benefits, which for most people means sick leave of more than 2 weeks. In addition, compared with national age-matched data, there were notably few deaths in the cohort [32]. This could perhaps be explained by the risk of people dying before being registered for sickness benefits for Covid-19, since the medical certificate for sick leave is usually written retrospectively on referral to a different hospital ward, for patients being treated in an intensive care unit.

About one-third of the patients were classified as "Covid-19, virus undetected", indicating insecurity in the data. Data collection was conducted in an early phase of the pandemic in Sweden, when PCR testing was limited. The authors found it most reasonable to consider the "Covid-19, virus undetected" diagnosis as a Covid-19 infection, to capture the picture of sick leave due to Covid-19. Furthermore, related diagnoses were merged into the Covid-19 sick-leave period if there was a gap shorter than 2 weeks. This decision was based on clinical experience and reasoning, to limit the risk of missing substantial sick leave due to difficulties of registration in the beginning of the pandemic. However, there is also a risk that a small number of the sick-leave periods were not due only to Covid-19. It was not possible to determine the exact prevalence of people suffering from symptoms of Covid-19 or long Covid in this study.

The ROC curves indicate that the accuracy of the regression models was low, so the predictions should be interpreted with caution. 


\section{Conclusion}

This comprehensive registry-based study showed that a substantial number of people are on sick leave due to Covid-19, and that the sick-leave period may be protracted. More than one out of ten people on sick leave were on it for more than 12 weeks (long Covid). This group of people seems to be heterogeneous and should not be neglected. Receiving inpatient care due to Covid19, being on sick leave prior to Covid-19, and older age all seemed to be associated with longer sick leave. The present findings are important for the health care and health insurance authorities.

\section{Abbreviations}

SSIA: Swedish Social Insurance Agency; ROC: Receiver Operating Characteristics; SD: Standard deviations; IQR: Interquartile range; OR: Odds ratio; Cl: Confidence interval

\section{Supplementary Information}

The online version contains supplementary material available at https://doi. org/10.1186/s12889-021-11013-2.

\section{Additional file 1.}

Additional file 2.

\section{Acknowledgments}

The authors would like to acknowledge the register holder, the Swedish Social Insurance Agency, the Swedish National Board of Health and Welfare, and Statistics Sweden for providing data. We would also like to thank our patient partner, Helena Strömberg, for her knowledgeable contribution to the research question and discussion of the results, and Adam Viktorisson for figure illustrations.

\section{Authors' contributions}

All authors were involved in conceptualisation and funding acquisition of the study. EW, AP and HCP were involved in data collection, and EW did the data handling, formal analysis, and were responsible for the figures. All authors were involved in data interpretation and manuscript writing. AP did the main literature search. The author(s) read and approved the final manuscript.

\section{Funding}

This study was founded by grants from the Swedish state under an agreement between the Swedish government and the county councils (ALF 73750, ALF 71980), AFA Insurance AFA 200324, Forte/Formas (2020-02775), and Regional research funding (VGFOUREG-940508) Vastragotaland region. Open Access funding provided by University of Gothenburg.

\section{Availability of data and materials}

The datasets used and/or analysed during the current study are available from the corresponding author on reasonable request. They are not publicly available, in accordance with the Ethics Review Authority. The study protocol and statistical analysis plan are available at https://www.researchweb.org/is/ vgr/project/274476.

\section{Declarations}

Ethics approval and consent to participate

The study has been approved by the Swedish Ethical Review Authority, Dnr: 2020-03046 and 2020-03922. The data have been handled in pseudonymised form, with the code key kept by the registry holders.

\section{Consent for publication}

Not applicable.

\section{Competing interests}

The authors declare that they have no competing interests.

Received: 15 March 2021 Accepted: 6 May 2021

Published online: 31 May 2021

\section{References}

1. Borg K, Stam H. Editorial: Covid-19 and Physical and Rehabilitation Medicine. J Rehabil Med. 2020;52(4):jrm00045.

2. Stam HJ, Stucki G, Bickenbach J. Covid-19 and Post Intensive Care Syndrome: A Call for Action. J Rehabil Med. 2020;52(4):jrm00044.

3. Brugliera L, Spina A, Castellazzi P, Cimino P, Tettamanti A, Houdayer E, et al. Rehabilitation of COVID-19 patients. J Rehabil Med. 2020;52(4):jrm00046.

4. Gutenbrunner C, Stokes EK, Dreinhofer K, Monsbakken J, Clarke S, Cote P, et al. Why Rehabilitation must have priority during and after the COVID-19pandemic: A position statement of the Global Rehabilitation Alliance. J Rehabil Med. 2020;52(7):jrm00081.

5. Helms J, Kremer S, Merdji H, Clere-Jehl R, Schenck M, Kummerlen C, et al. Neurologic features in severe SARS-CoV-2 infection. N Engl J Med. 2020; 382(23):2268-70. https://doi.org/10.1056/NEJMc2008597.

6. Khan F, Amatya B. Medical Rehabilitation in Pandemics: Towards a New Perspective. J Rehabil Med. 2020;52(4):jrm00043.

7. Calvo-Bonacho E, Catalina-Romero C, Fernández-Labandera C, FernándezMeseguer A, González-Quintela A, Martínez-Muñoz P, et al. COVID-19 and Sick Leave: An Analysis of the Ibermutua Cohort of Over 1,651,305 Spanish Workers in the First Trimester of 2020. Front Public Health. 2020:8(590):1-6.

8. Heymann J, Raub A, Waisath W, McCormack M, Weistroffer R, Moreno G, et al. Protecting health during COVID-19 and beyond: a global examination of paid sick leave design in 193 countries*. Global Public Health. 2020;15(7): 925-34. https://doi.org/10.1080/17441692.2020.1764076.

9. Försäkringskassan (Swedish Social Insurance Agency): Rapport - Effekter som covid-19 har på sjukförsäkringen. In. Edited by Försäkringskassan, vol. 1; 2021: 82 .

10. Covid-19 (inkl de patienter som fått intensivvård med respiratorbehandling) [https://roi.socialstyrelsen.se/fmb/COVID-19-inkl-de-patienter-som-fattintensivvard-med-respiratorbehandling/702].

11. Carvalho-Schneider C, Laurent E, Lemaignen A, Beaufils E, Bourbao-Tournois C, Laribi S, et al. Follow-up of adults with noncritical COVID-19 two months after symptom onset. Clin Microbiol Infect. 2021;27(2):258-63. https://doi. org/10.1016/j.cmi.2020.09.052.

12. Davis HE, Assaf GS, McCorkell L, Wei H, Low RJ, Re'em Y, et al. Characterizing Long COVID in an International Cohort: 7 Months of Symptoms and Their Impact. medRxiv. 2020:2020.2012.2024.20248802.

13. Michelen M, Manoharan L, Elkheir N, Cheng V, Dagens D, Hastie C, et al. Characterising long-term covid-19: a rapid living systematic review. medRxiv. 2020:2020.2012.2008.20246025

14. Sudre CH, Murray B, Varsavsky T, Graham MS, Penfold RS, Bowyer RC, et al. Attributes and predictors of long COVID. Nat Med. 2021;27(4):626-31.

15. Huang C, Huang L, Wang Y, Li X, Ren L, Gu X, et al. 6-month consequences of COVID-19 in patients discharged from hospital: a cohort study. Lancet. 2021;397(10270):220-32. https://doi.org/10.1016/S0140-6736(20)32656-8.

16. World Health Organization: In the wake of the pandemic: Preparing for Long COVID. In. Edited by Sagan A; 2021: 1-29.

17. World Health Organization. International statistical classification of diseases and related health problems. 10th revision ed. Geneva: World Health Organization; 1992

18. Field A. Discovering statistics using IBM SPSS statistics : and sex and drugs and rock 'n' roll. 4th ed. Los Angeles, London: Sage; 2013.

19. Lew HL, Oh-Park M, Cifu DX. The war on COVID-19 pandemic: role of rehabilitation professionals and hospitals. Am J Phys Med Rehabil. 2020; 99(7):571-2. https://doi.org/10.1097/PHM.0000000000001460.

20. Herridge MS, Tansey CM, Matte A, Tomlinson G, Diaz-Granados N, Cooper A, et al. Functional disability 5 years after acute respiratory distress syndrome. $\mathrm{N}$ Engl J Med. 2011;364(14):1293-304. https://doi.org/10.1056/NEJMoa1011802.

21. Haitao T, Vermunt JV, Abeykoon J, Ghamrawi R, Gunaratne M, Jayachandran M, et al. COVID-19 and sex differences: mechanisms and biomarkers. Mayo Clin Proc. 2020;95(10):2189-203. https://doi.org/10.1016/j.mayocp.2020.07.024.

22. Public Health Agency of Sweden [https://www.folkhalsomyndigheten.se/ smittskydd-beredskap/utbrott/aktuella-utbrott/COVID-19/statistik-och-ana lyser/bekraftade-fall-i-sverige/]. 
23. Jiang Z, Dreyer RP, Spertus JA, Masoudi FA, Li J, Zheng X, et al. Factors associated with return to work after acute myocardial infarction in China. JAMA Netw Open. 2018;1(7):e184831. https://doi.org/10.1001/jama networkopen.2018.4831.

24. Street TD, Lacey SJ. A systematic review of studies identifying predictors of poor return to work outcomes following workplace injury. Work. 2015;51(2): 373-81. https://doi.org/10.3233/WOR-141980.

25. Westerlind E, Persson HC, Sunnerhagen KS. Return to work after a stroke in working age persons; a six-year follow up. PLoS One. 2017;12(1):e0169759. https://doi.org/10.1371/journal.pone.0169759.

26. Larsson J, Esbjornsson E, Bjorkdahl A, Morberg I, Nilsson M, Sunnerhagen KS. Sick leave after traumatic brain injury. The person or the diagnosis--which has greater impact? Scand J Public Health. 2010;38(5):541-7. https://doi. org/10.1177/1403494810371143.

27. Sakakibara S, Sado M, Ninomiya A, Arai M, Takahashi S, Ishihara C, et al. Predictive factors of the duration of sick leave due to mental disorders. Int J Ment Health Syst. 2019;13(1):19. https://doi.org/10.1186/s13033-019-0279-6.

28. Chen Y, Klein SL, Garibaldi BT, Li H, Wu C, Osevala NM, et al. Aging in COVID-19: vulnerability, immunity and intervention. Ageing Res Rev. 2021; 65:101205. https://doi.org/10.1016/j.arr.2020.101205.

29. Raisi-Estabragh Z, McCracken C, Bethell MS, Cooper J, Cooper C, Caulfield MJ, et al. Greater risk of severe COVID-19 in black, Asian and minority ethnic populations is not explained by cardiometabolic, socioeconomic or behavioural factors, or by $25(\mathrm{OH})$-vitamin D status: study of 1326 cases from the UK biobank. J Public Health (Oxf). 2020;42(3):451-60. https://doi.org/10.1 093/pubmed/fdaa095

30. Dorn AV, Cooney RE, Sabin ML. COVID-19 exacerbating inequalities in the US. Lancet. 2020;395(10232):1243-4. https://doi.org/10.1016/S0140-6736(20)3 0893-X.

31. Burstrom B, Tao W. Social determinants of health and inequalities in COVID-19. Eur J Pub Health. 2020;30(4):617-8. https://doi.org/10.1093/eurpub/ckaa095.

32. [https://www.socialstyrelsen.se/statistik-och-data/statistik/statistik-om-covid-1 9/statistik-over-antal-avlidna-i-covid-19/].

\section{Publisher's Note}

Springer Nature remains neutral with regard to jurisdictional claims in published maps and institutional affiliations.

Ready to submit your research? Choose BMC and benefit from:

- fast, convenient online submission

- thorough peer review by experienced researchers in your field

- rapid publication on acceptance

- support for research data, including large and complex data types

- gold Open Access which fosters wider collaboration and increased citations

- maximum visibility for your research: over $100 \mathrm{M}$ website views per year

At $\mathrm{BMC}$, research is always in progress.

Learn more biomedcentral.com/submissions 